\title{
Viscous generalized Chaplygin gas as a unified dark fluid: including perturbation of bulk viscosity
}

\author{
Wei $\mathbf{L i}^{1,2, a}$, Lixin $\mathbf{X u}^{1, b}$ \\ ${ }^{1}$ Institute of Theoretical Physics, School of Physics and Optoelectronic Technology, Dalian University of Technology, Dalian 116024, \\ People's Republic of China \\ ${ }^{2}$ Department of Physics, College of Mathematics and Physics, Bohai University, Jinzhou 121013, People's Republic of China
}

Received: 3 November 2013 / Accepted: 30 November 2013 / Published online: 20 February 2014

(C) The Author(s) 2014. This article is published with open access at Springerlink.com

\begin{abstract}
In this paper, we continue our previous work of studying viscous generalized Chaplygin gas as a unified dark fluid but the bulk viscosity perturbations. By using the currently available cosmic observational data from SNLS3, BAO, HST, and recently released Planck, we obtain a constraint on the bulk viscosity coefficient: $\zeta_{0}=$ $0.0000138_{-0.0000105-0.0000138-0.0000138}^{+0.00000614+0.0000145+0.0000212}$ in $1,2,3 \sigma$ regions, respectively, via the Markov Chain Monte Carlo method. The result shows that when considering perturbations of the bulk viscosity, the current cosmic observations favor a smaller bulk viscosity coefficient.
\end{abstract}

\section{Introduction}

Several astronomical observations (such as high-redshift supernovae of type Ia (SN Ia) [1-5], cosmic microwave background (CMB) radiation [6], matter power spectra [7], etc.) strongly suggest that at present about $96 \%$ of the cosmological total energy content is dynamically dominated by a dark sector which is responsible for the acceleration of our universe. This dark sector is generally assumed to have two different components: dark energy and dark matter. To investigate this dark sector, many cosmological models have been built based on the dynamics governed by Einstein's general relativity. Validity of the cosmological principle at large cosmic scales is assumed and also that the medium of the universe can be modeled as an idealized perfect fluid; this means that all components of the matter-energy in our universe are considered as a perfect fluid without any viscosity or it may be interpreted as the result of the gross grain approximation having been taken. The most natural dark energy candidate is a cosmological constant which arises as the result of a

\footnotetext{
a e-mail: liweizhd@126.com

be-mail: 1xxu@dlut.edu.cn
}

combination of quantum field theory and general relativity. But research has shown that constant dark energy models are not well confirmed by observations nor by theoretical considerations $[32,33]$. One of the alternatives to the cosmological constant is to describe dark matter and dark energy within a unified dark fluid model. To the best of our knowledge, the first proposal along this line was the Chaplygin gas [8-10] in its original and generalized forms [11-14]. However, the unified Chaplygin gas type models, in spite of many attractive features, seemed to suffer from a major drawback: it predicted strong small scale oscillations or instabilities in the matter power spectrum, in complete disagreement with the observational data [15]. These apparently unrealistic predictions are the result of an adiabatic perturbation analysis. It has been suggested that non-adiabatic perturbations may alleviate or even avoid this problem $[16,17,28]$. A reasonable possibility is to allow the generalized Chaplygin gas to have non-adiabatic perturbations, which is a natural assumption, since it is not a pressureless fluid actually. An attempt in this direction has already been performed in [30] and [31]. Furthermore, in recent years, more and more cosmological observations have suggested that our universe is permeated by an imperfect fluid, in which we have a negative pressure, as was argued in [28,29]; an effective pressure including bulk viscosity might play the role of the agent that drives the present acceleration of universe.

The viscous generalized Chaplygin gas (hereafter referred to as VGCG) is a widely studied model among those proposed to describe the observed accelerated expansion of the universe. In contrast to many models describing dark energy alone, the VGCG gives a unified description of dark matter and dark energy, enrolling itself in the class of so-called unified dark fluid (UDF) cosmological models see e.g. [18-22]. A common characteristic of these papers is that only the impact of the bulk viscosity on the background expansion of the universe is studied without considering perturbations 
of the bulk viscosity. However, the perturbation analysis of the viscous cosmological models is crucially important to the evolution of cosmology. The different approaches mentioned imply a generally different dynamics at the perturbative level. The unifying view on the background level is accompanied by a difference in the perturbation dynamics. This circumstance renders the different approaches potentially testable alternatives to the $\triangle \mathrm{CDM}$ model while sharing the same background dynamics of the latter. Therefore, it is interesting to study the behavior of the VGCG under perturbations. The cosmological perturbation issue was first tackled and studied by Lifshitz in 1946 in the synchronous gauge [23,24]. Subsequently in 1980 Bardeen developed gauge-invariant perturbation theory [25] through constructing suitable combinations of metric and stress-energy tensor perturbations which are invariant under a generic gauge transformation. The gaugeinvariant formalism has been used and reviewed by many authors.

In the present paper, we follow the notation of [26] and study only scalar perturbations. We will describe the bulk viscous pressure by Eckart's expression [34], $p_{v}=-\zeta u_{; \mu}^{\mu}$, where the (non-negative) quantity $\zeta$ is the (generally not constant) bulk viscosity coefficient and $u_{; \mu}^{\mu}$ is the fluid-expansion scalar, which in a homogeneous and isotropic background reduces to $3 H$, where $H=\frac{\dot{a}}{a}$ is the Hubble parameter and $a$ is the scale factor of the Robertson-Walker metric. As a continuation of our previous work [27], here we investigate the VGCG model by including a bulk viscosity perturbation.

The structure of this paper is as follows. In the next section, we briefly introduce some basic equations of the viscous generalized Chaplygin gas model. The derivation of the evolution equations for the density perturbation and the velocity perturbations are presented in Sect. 3. Then in Sect. 4, by using the MCMC method, we perform a global fitting to the current observational data and analyze the constraint results. The discussion and conclusion are in the final section.

\section{Basic equations of viscous generalized Chaplygin gas model}

In an isotropic and homogeneous universe, we consider the standard Friedmann-Robertson-Walker metric,

$\mathrm{d} s^{2}=-\mathrm{d} t^{2}+a^{2}(t)\left[\frac{1}{1-k r^{2}} \mathrm{~d} r^{2}+r^{2}\left(\mathrm{~d} \theta^{2}+\sin ^{2} \theta \mathrm{d} \phi^{2}\right)\right]$.

For the sake of simplicity, we choose the flat geometry $k=0$, which is also favored by the update result of the cosmic background radiation measurement. The general stress-energymomentum tensor is

$T_{\mu \nu}=(\rho+p) U_{\mu} U_{\nu}+p g_{\mu \nu}$.
To consider the effect of bulk viscosity, we modify the pressure only by redefining the effective pressure $p_{\text {eff }}$, according to $p_{\text {eff }}=p+p_{v}=p-3 H \zeta$, and we re-write the viscous energy-momentum tensor [35] as

$$
\begin{aligned}
T_{\text {viscous }}^{\mu \nu} & =\rho U^{\mu} U^{v}+\left(p-3 \zeta \frac{\dot{a}}{a}\right)\left(g^{\mu \nu}+U^{\mu} U^{v}\right) \\
& \equiv p_{\text {eff }} g^{\mu \nu}+\left(p_{\text {eff }}+\rho\right) U^{\mu} U^{v} .
\end{aligned}
$$

From the equation above, we see that the effect of the bulk viscosity is to change the pressure $p$ to an effective pressure $p_{\text {eff }}=p-3 \zeta \dot{a} / a$. The physical interpretation is clear: the viscous pressure can play the role of an agent that drives the present acceleration of the universe. Note that the possibility of a viscosity dominated late epoch of the universe with accelerated expansion was already mentioned by Padmanabhan and Chitre in [36].

Using the GCG equation of state $p_{g}=-A / \rho_{g}^{\alpha}$, which yields an analytically solvable cosmological dynamics if the universe is GCG dominated, we obtain the equation of state (EoS) of the viscous GCG (VGCG) model as given in the form of

$p_{\mathrm{VGCG}}=-A / \rho_{\mathrm{VGCG}}^{\alpha}-3 H \zeta$.

This EoS includes the GCG model as a special case when $\zeta=0$; when $\zeta \neq 0$, for the normal form $\zeta=\zeta_{0} \rho_{\mathrm{VGCG}}^{\frac{1}{2}}$, we have the EoS

$p_{\mathrm{VGCG}}=-A / \rho_{\mathrm{VGCG}}^{\alpha}-\sqrt{3} \zeta_{0} \rho \mathrm{VGCG}$,

where $A, \zeta_{0}$ and $\alpha$ are model parameters. Applying energy conservation of VGCG, one can deduce its energy density as

$$
\begin{aligned}
\rho_{\mathrm{VGCG}}= & \rho_{\mathrm{VGCG} 0}\left[\frac{B_{s}}{1-\sqrt{3} \zeta_{0}}+\left(1-\frac{B_{s}}{1-\sqrt{3} \zeta_{0}}\right)\right. \\
& \left.\times a^{-3(1+\alpha)\left(1-\sqrt{3} \zeta_{0}\right)}\right]^{\frac{1}{1+\alpha}},
\end{aligned}
$$

where $B_{s}=A / \rho_{\mathrm{VGCG} 0}^{1+\alpha}, \alpha$ and $\zeta_{0}$ are model parameters. From Eq. (6) one finds that $0 \leq B_{s} \leq 1$ and $\zeta_{0}<\frac{1}{\sqrt{3}}$ are required to keep positivity of the energy density. If $\alpha=0$ and $\zeta_{0}=0$ in Eq. (6), the standard $\triangle \mathrm{CDM}$ model is recovered. Taking VGCG as a unified component, one has the Friedmann equation

$$
\begin{aligned}
H^{2}= & H_{0}^{2}\left\{( 1 - \Omega _ { b } - \Omega _ { r } - \Omega _ { k } ) \left[\frac{B_{s}}{1-\sqrt{3} \zeta_{0}}\right.\right. \\
& \left.+\left(1-\frac{B_{s}}{1-\sqrt{3} \zeta_{0}}\right) a^{-3(1+\alpha)\left(1-\sqrt{3} \zeta_{0}\right)}\right]^{\frac{1}{1+\alpha}} \\
& \left.+\Omega_{b} a^{-3}+\Omega_{r} a^{-4}+\Omega_{k} a^{-2}\right\}
\end{aligned}
$$

where $H$ is the Hubble parameter with its current value $H_{0}=100 \mathrm{~h} \mathrm{~km} \mathrm{~s}^{-1} \mathrm{Mpc}^{-1}$, and $\Omega_{i}(i=b, r, k)$ are dimensionless energy parameters of baryon, radiation, and effec- 
tive curvature density, respectively. In this paper, we only consider the spatially flat FRW universe.

Here, we treat VGCG as a unified dark fluid which interacts with the remaining matter purely through gravity. With the assumption of a pure adiabatic contribution to the perturbations, the adiabatic sound speed for VGCG is

$c_{s, a d}^{2}=\frac{\dot{p}_{\mathrm{VGCG}}}{\dot{\rho}_{\mathrm{VGCG}}}=-\alpha w_{\mathrm{eff}}-\sqrt{3} \zeta_{0}$,

where $w_{\text {eff }}$ is the EoS of VGCG in the form of

$w_{\text {eff }}=-\frac{B_{s}}{B_{s}+\left(1-B_{s}\right) a^{-3(1+\alpha)}}-\sqrt{3} \zeta_{0}$.

From the above equation, one can find that in order to protect the sound of speed from negativity, $\alpha \geq 0$ is required because of the non-positive values of $w_{\text {eff }}$.

We studied the perturbation evolution equations of VGCG in order to study the effects on the CMB anisotropic power spectrum. In the synchronous gauge, using conservation of the energy-momentum tensor, $T_{\text {(viscous) }}^{\mu} \underset{v ; \mu}{\mu}=0$, one has the perturbation equations of the density contrast and the velocity divergence for the VGCG

$$
\begin{aligned}
\dot{\delta}_{\mathrm{VGCG}}= & -\left(1+w_{\mathrm{eff}}\right)\left(\theta_{\mathrm{VGCG}}+\frac{\dot{h}}{2}\right) \\
& -3 \mathcal{H}\left(c_{s}^{2}-w_{\mathrm{eff}}\right) \delta_{\mathrm{VGCG}}, \\
\dot{\theta}_{\mathrm{VGCG}}= & -\mathcal{H}\left(1-3 c_{s}^{2}\right) \theta_{\mathrm{VGCG}}+\frac{c_{s}^{2}}{1+w_{\mathrm{eff}}} k^{2} \delta_{\mathrm{VGCG}} \\
& -k^{2} \sigma_{\mathrm{VGCG}},
\end{aligned}
$$

following the notation of $\mathrm{Ma}$ and Bertschinger [37]. For the perturbation theory in a gauge-ready formalism, please see [38]. For the shear perturbation $\sigma_{\mathrm{VGCG}}=0$ is assumed and adiabatic initial conditions are adopted in our calculation. When the EoS of a pure barotropic fluid is negative, it has an imaginary adiabatic sound speed, which causes instability of the perturbations; for example, the $w=$ constant quintessence dark energy model. The way to overcome this problem is to allow an entropy perturbation and to assume a positive or null effective speed of sound; of this we will give a detailed study in the following.

\section{Perturbation equations}

\subsection{Perturbed metric and energy-momentum tensor}

Scalar perturbations of the flat FRW metric are given in the following form:

$$
\begin{aligned}
\mathrm{d} s^{2}= & a^{2}\left\{-(1+2 \phi) \mathrm{d} \tau^{2}+2 \partial_{i} B \mathrm{~d} \tau \mathrm{d} x^{i}\right. \\
& \left.+\left[(1-2 \psi) \delta_{i j}+2 \partial_{i} \partial_{j} E\right] \mathrm{d} x^{i} \mathrm{~d} x^{j}\right\},
\end{aligned}
$$

where $a$ is the scale factor, $\tau$ is the conformal time, $x_{i}$ are the spatial coordinates and $\phi$ and $\psi$ are the metric perturbations. The background four-velocity is $\bar{u}^{\mu}=a^{-1}(1,0,0,0)$, which can be derived as follows:

$\bar{u}^{\mu}=-\frac{\mathrm{d} \tau}{\mathrm{d} s}=-\frac{1}{a} \frac{\mathrm{d} t}{\mathrm{~d} s}=\frac{1}{a} \delta_{0}^{\mu}$.

The spatial part is the perturbation; we can set it as $\partial^{i} v$ for scalar perturbations only. Then using the equality $g_{\mu \nu} u^{\mu} u^{\nu}=-1$, one has

$u^{0}= \pm \frac{1}{\sqrt{g_{00}}}=+\frac{1}{a}(1-\phi)$,

so one has the following four-velocity of the fluid:

$u^{\mu}=a^{-1}\left(1-\phi, \partial^{i} v\right)$,

$u_{\mu}=g_{\mu \nu} u^{\nu}=a\left(-1-\phi, \partial_{i}[v+B]\right)$,

where $v$ is the peculiar velocity potential. The local volume expansion rate is $\theta=\vec{\nabla} \cdot \vec{v}$. Then one has the expansion rate $\theta=-k^{2}(v+B)$ for the fluid. Let $u^{\mu}$ be the energyframe four-velocity (zero momentum flux relative to $u^{\mu}$ ). The energy density is the eigenvalue for this four-velocity, i.e., $T_{v}^{\mu} u^{\mu}=-\rho u^{\mu}$. The energy-momentum tensor can be written as

$T_{v}^{\mu}=\left(\rho+p_{\mathrm{eff}}\right) u^{\mu} u_{v}+p_{\mathrm{eff}} \delta_{v}^{\mu}$,

where $\rho=\bar{\rho}+\delta \rho$ and $p=\bar{p}+\delta p$. The effective pressure $p_{\text {eff }}$ is given as

$p_{\text {eff }}=p-\zeta \overline{\left(\nabla_{\gamma} u^{\gamma}\right)}=p-\frac{3 \mathcal{H}}{a} \zeta$.

The general energy-momentum tensor is

$T_{0}^{0}=-\bar{\rho}-\delta \rho$,

$T_{i}^{0}=\left(\bar{\rho}+\overline{p_{\text {eff }}}\right) \partial_{i}(v+B)=\left(\bar{\rho}+\overline{p_{\text {eff }}}\right)\left(v_{i}+B_{i}\right)$,

$T_{0}^{i}=-\left(\bar{\rho}+\overline{p_{\mathrm{eff}}}\right) v^{i}$,

$T_{j}^{i}=\overline{p_{\mathrm{eff}}} \delta_{j}^{i}+\delta p_{\mathrm{eff}} \delta_{j}^{i}$.

Then one has the background energy-momentum tensor

$\overline{T_{0}^{0}}=-\bar{\rho}, \quad \overline{T_{i}^{0}}=0, \quad \overline{T_{0}^{i}}=0, \quad \overline{T_{j}^{i}}=\overline{p_{\mathrm{eff}}} \delta_{j}^{i}$.

Thus the perturbed energy-momentum tensor can be written as

$\delta T_{0}^{0}=-\delta \rho, \quad \delta T_{i}^{0}=\left(\bar{\rho}+\overline{p_{\text {eff }}}\right)\left(v_{i}+B_{i}\right)$,

$\delta T_{0}^{i}=-\left(\bar{\rho}+\overline{p_{\text {eff }}}\right) v^{i}, \quad \delta T_{j}^{i}=\delta p_{\text {eff }} \delta_{j}^{i}$.

\subsection{Calculation of Christoffel symbols}

The formula of the Christoffel symbols is

$\Gamma_{\alpha \beta}^{\mu}=\frac{1}{2} g^{\mu \nu}\left(g_{\alpha \nu, \beta}+g_{\beta \nu, \alpha}-g_{\alpha \beta, v}\right)$, 
where the comma in the index stands for the derivative, and Greek letters $\mu, \nu, \alpha, \beta$ take the values $0,1,2,3$. In the following, the prime stands for the derivative with respect to the conformal time $\tau$. So one has the following equations:

$$
\begin{aligned}
\Gamma_{00}^{0}= & \mathcal{H}+\phi^{\prime}, \\
\Gamma_{0 i}^{0}= & \phi_{i}+\mathcal{H} B_{i}, \\
\Gamma_{i j}^{0}= & \mathcal{H} \delta_{i j}-\left[\psi^{\prime}+2 \mathcal{H}(\psi+\phi)\right] \delta_{i j} \\
& +\partial_{i} \partial_{j}\left(E^{\prime}-B+2 \mathcal{H} E\right), \\
\Gamma_{00}^{i}= & \partial^{i}\left(\phi+B^{\prime}+\mathcal{H} B\right), \\
\Gamma_{j 0}^{i}= & \mathcal{H} \delta_{j}^{i}-\psi^{\prime} \delta_{j}^{i}+\partial_{j} \partial^{i} E^{\prime}, \\
\Gamma_{j k}^{i}= & -\mathcal{H} \partial^{i} B \delta_{j k}+\delta_{j k} \partial^{i} \psi-\delta_{j}^{i} \partial_{k} \psi \\
& -\delta_{k}^{i} \partial_{j} \psi+\partial_{j} \partial_{k} \partial^{i} E .
\end{aligned}
$$

The nonzero Christoffel symbols are shown in the following; the background items are

$\bar{\Gamma}_{00}^{0}=\mathcal{H}, \quad \bar{\Gamma}_{i j}^{0}=\mathcal{H} \delta_{i j}, \quad \bar{\Gamma}_{j 0}^{i}=\mathcal{H} \delta_{j}^{i}$,

the perturbed items are

$$
\begin{aligned}
\delta \Gamma_{00}^{0}= & \phi^{\prime}, \quad \delta \Gamma_{00}^{i}=\partial^{i}\left(\phi+B^{\prime}+\mathcal{H} B\right) \\
\delta \Gamma_{0 i}^{0}= & \partial_{i} \phi+\mathcal{H} \partial_{i} B, \delta \Gamma_{j 0}^{i}=-\psi^{\prime} \delta_{j}^{i}+\partial_{j} \partial^{i} E^{\prime} \\
\delta \Gamma_{i j}^{0}= & -\left[\psi^{\prime}+2 \mathcal{H}(\phi+\psi)\right] \delta_{i j}+\partial_{i} \partial_{j}\left[E^{\prime}+2 \mathcal{H} E-B\right] \\
\delta \Gamma_{j k}^{i}= & -\mathcal{H} \partial^{i} B \delta_{j k}+\delta_{j k} \partial^{i} \psi-\delta_{j}^{i} \partial_{k} \psi-\delta_{k}^{i} \partial_{j} \psi \\
& +\partial_{j} \partial_{k} \partial^{i} E
\end{aligned}
$$

\subsection{Evolution equations for density perturbation} and velocity perturbations

In this section, we will given a derivation of the perturbed energy-momentum equations. From the formulas

$$
\begin{aligned}
& \delta \nabla_{\mu} T^{\mu 0}=\delta g^{\mu \sigma} \overline{\nabla_{\mu} T_{\sigma}^{0}}+\bar{g}^{\mu \sigma} \delta \nabla_{\mu} T_{\sigma}^{0}, \\
& \delta \nabla_{\mu} T^{\mu i}=\delta g^{\mu \sigma} \overline{\nabla_{\mu} T_{\sigma}^{i}}+\bar{g}^{\mu \sigma} \delta \nabla_{\mu} T_{\sigma}^{i},
\end{aligned}
$$

and

$$
\begin{aligned}
& \nabla_{\mu} T_{\sigma}^{v}=T_{\sigma, \mu}^{v}+\Gamma_{\rho \mu}^{v} T_{\sigma}^{\rho}-\Gamma_{\sigma \mu}^{\rho} T_{\rho}^{v}, \\
& \bar{\nabla}_{\mu} T_{\sigma}^{v}=\bar{T}_{\sigma, \mu}^{v}+\bar{\Gamma}_{\rho \mu}^{v} \bar{T}_{\sigma}^{\rho}-\bar{\Gamma}_{\sigma \mu}^{\rho} \bar{T}_{\rho}^{v},
\end{aligned}
$$

namely

$$
\begin{aligned}
& \overline{\nabla_{0} T_{0}^{0}}=-\bar{\rho}^{\prime}, \quad \delta \nabla_{0} T_{0}^{0}=-\delta \rho^{\prime}, \\
& \overline{\nabla_{0} T_{i}^{0}}=0, \quad \overline{\nabla_{i} T_{j}^{0}}=\mathcal{H}\left(\bar{\rho}+\overline{p_{\text {eff }}}\right) \delta_{i j}, \\
& \overline{\nabla_{i} T_{0}^{0}}=0, \quad \delta \nabla_{i} T_{0}^{0}=-\mathcal{H}\left(\bar{\rho}+\overline{p_{\text {eff }}}\right)\left(2 v_{i}+B_{i}\right), \\
& \delta \nabla_{0} T_{i}^{0}=\left[\left(\bar{\rho}+\overline{p_{\text {eff }}}\right)\left(v_{i}+B_{i}\right)\right]^{\prime} \\
& +\left(\bar{\rho}+\overline{p_{\text {eff }}}\right)\left(\partial_{i} \phi+\mathcal{H} \partial_{i} B\right), \\
& \delta \nabla_{i} T_{j}^{0}=\left(\bar{\rho}+\overline{p_{\text {eff }}}\right)\left(\partial_{i} \partial_{j} v+\partial_{i} \partial_{j} B\right)+\mathcal{H}\left(\delta \rho+\delta p_{\text {eff }}\right) \delta_{i j} \\
& -\left(\bar{\rho}+\overline{p_{\text {eff }}}\right)\left[\psi^{\prime}+2 \mathcal{H}(\psi+\phi)\right] \delta_{i j} \\
& +\left(\bar{\rho}+\overline{p_{\text {eff }}}\right) \nabla^{2}\left(E^{\prime}+2 \mathcal{H} E-B\right),
\end{aligned}
$$

we obtain the following perturbed energy-momentum equation:

$$
\begin{gathered}
\delta \nabla_{\mu} T^{\mu 0}=\frac{1}{a^{2}}\left\{\delta \rho^{\prime}+3 \mathcal{H}\left(\delta \rho+\delta p_{\text {eff }}\right)-3\left(\bar{\rho}+\overline{p_{\text {eff }}}\right) \psi^{\prime}\right. \\
\left.+\left(\bar{\rho}+\overline{p_{\text {eff }}}\right) \nabla^{2}\left(v+E^{\prime}\right)-2 \phi\left[\bar{\rho}^{\prime}+3 \mathcal{H}\left(\bar{\rho}+\overline{p_{\text {eff }}}\right)\right]\right\} .
\end{gathered}
$$

And in the same way, we make use of the following results:

$$
\begin{aligned}
\overline{\nabla_{0} T_{0}^{i}=} & 0, \quad \overline{\nabla_{j} T_{0}^{i}}=-\mathcal{H}\left(\bar{\rho}+\overline{p_{\mathrm{eff}}}\right) \delta_{j}^{i}, \\
\delta \nabla_{0} T_{0}^{i}= & -\left[\left(\bar{\rho}+\overline{p_{\mathrm{eff}}}\right) v^{i}\right]^{\prime} \\
& -\left(\bar{\rho}+\overline{p_{\mathrm{eff}}}\right) \partial^{i}(\phi+B+\mathcal{H} B), \\
\overline{\nabla_{0} T_{k}^{i}}= & p_{\mathrm{eff}}^{\prime} \delta_{k}^{i}, \delta \nabla_{0} T_{k}^{i}=\delta p_{\mathrm{eff}}^{\prime} \delta_{k}^{i}, \\
\overline{\nabla_{j} T_{k}^{i}}= & \partial_{j}\left(\overline{p_{\mathrm{eff}}} \delta_{k}^{i}\right), \\
\delta \nabla_{j} T_{k}^{i}= & \mathcal{H}\left(\bar{\rho}+\overline{p_{\mathrm{eff}}}\right)\left[\delta_{j}^{i} \partial_{k} v+\delta_{j}^{i} \partial_{k} B+\delta_{k j} \partial^{i} v\right] \\
& +\partial_{j}\left(\delta p_{\mathrm{eff}} \delta_{k}^{i}\right), \\
\delta \nabla_{j} T_{0}^{i}= & -\left(\bar{\rho}+\overline{p_{\mathrm{eff}}}\right)\left(-\psi^{\prime} \delta_{j}^{i}+\partial^{i} \partial_{j} E^{\prime}\right) \\
& -\mathcal{H} \delta_{j}^{i}\left(\delta \rho+\delta p_{\mathrm{eff}}\right)-\left(\bar{\rho}+\overline{p_{\mathrm{eff}}}\right) \partial_{j} \partial^{i} v,
\end{aligned}
$$

and we also have the following equation:

$$
\begin{aligned}
\delta \nabla_{\mu} T^{\mu i}= & \frac{1}{a^{2}} \partial^{i}\left\{\left[\left(\bar{\rho}+\overline{p_{\mathrm{eff}}}\right)(v+B)\right]^{\prime}+\left(\bar{\rho}+\overline{p_{\mathrm{eff}}}\right) \phi\right. \\
& +4 \mathcal{H}\left(\bar{\rho}+\overline{p_{\mathrm{eff}}}\right)(v+B)+\delta p_{\mathrm{eff}} \\
& \left.-\left[\bar{\rho}^{\prime}+3 \mathcal{H}\left(\bar{\rho}+\overline{p_{\mathrm{eff}}}\right)\right] B\right\} .
\end{aligned}
$$

If the fluid is conserved, i.e. $\bar{\rho}^{\prime}+3 \mathcal{H}\left(\bar{\rho}+\overline{p_{\text {eff }}}\right)=0$, the above perturbed equations can be rewritten as

$$
\begin{aligned}
\delta \nabla_{\mu} T^{\mu 0}= & \frac{1}{a^{2}}\left\{\delta \rho^{\prime}+3 \mathcal{H}\left(\delta \rho+\delta p_{\text {eff }}\right)-3\left(\bar{\rho}+\overline{p_{\text {eff }}}\right) \psi^{\prime}\right. \\
& \left.+\left(\bar{\rho}+\overline{p_{\text {eff }}}\right) \nabla^{2}\left(v+E^{\prime}\right)\right\}, \\
\delta \nabla_{\mu} T^{\mu i}= & \frac{1}{a^{2}} \partial^{i}\left\{\left[\left(\bar{\rho}+\overline{p_{\text {eff }}}\right)(v+B)\right]^{\prime}+\left(\bar{\rho}+\overline{p_{\text {eff }}}\right) \phi\right. \\
& \left.+4 \mathcal{H}\left(\bar{\rho}+\overline{p_{\text {eff }}}\right)(v+B)+\delta p_{\text {eff }}\right\}
\end{aligned}
$$

where $\overline{p_{\mathrm{eff}}}=\bar{p}-\frac{3}{a} \mathcal{H} \zeta$

$$
\begin{aligned}
\delta p_{\text {eff }} & =\delta p-\delta \zeta \overline{\left(\nabla_{\gamma} u^{\gamma}\right)}-\zeta\left(\delta \nabla_{\gamma} u^{\gamma}\right) \\
& =\delta p-\frac{3 \mathcal{H}}{a} \delta \zeta-\frac{\zeta}{a}\left[\nabla^{2}\left(v+E^{\prime}\right)-\left(3 \psi^{\prime}+3 \mathcal{H} \phi\right)\right] .
\end{aligned}
$$

To solve the above equations or make them complete, we need the relations between $\delta p$ and $\delta \rho$. The sound speed $c_{s \text {,eff }}^{2}$ of a fluid or scalar field is the propagation speed of a pressure fluctuation in the rest frame,

$c_{s, \mathrm{eff}}^{2}=\left.\frac{\delta p_{\mathrm{eff}}}{\delta \rho}\right|_{r f}$, 
where ' $\left.\right|_{r f}$ ' denotes the rest frame. For a scalar field $\phi$, the rest frame is defined as the hypersurface $\delta \phi=0$, i.e. $\phi=$ constant. So, one has $\delta V=0$ and $\delta \rho_{\phi}=\delta\left(\frac{1}{2} a^{-2} \phi^{\prime 2}+V\right)=$ $a^{-2} \phi^{\prime} \delta \phi^{\prime}=\delta p_{\phi}$. Thus the sound speed of the scalar field equals the speed of light and is independent from the form of $V(\phi)$,

$\left.\delta \phi\right|_{r f}=0 \Rightarrow c_{s \phi}^{2}=1$.

The 'adiabatic sound speed' for any medium is defined as

$c_{a, \mathrm{eff}}^{2}=\frac{p_{\mathrm{eff}}^{\prime}}{\rho^{\prime}}=w_{\mathrm{eff}}+\frac{w_{\mathrm{eff}}^{\prime}}{\rho^{\prime} / \rho}$.

The rest frame (the zero momentum gauge or comoving orthogonal gauge) is the comoving $\left(\left.v\right|_{r f}=0\right)$ orthogonal $\left.\left(\left.B\right|_{r f}\right)=0\right)$ frame, so that

$T_{0}^{i}\left|r f=0=T_{i}^{0}\right| r f$.

We make a gauge transformation, $x^{\mu} \rightarrow x^{\mu}+\left(\delta \tau, \partial^{i} \delta x\right)$, from the rest frame gauge to a general gauge,

$v+B=\left.(v+B)\right|_{r f}+\delta \tau, \quad \delta p=\left.\delta p\right|_{r f}-p^{\prime} \delta \tau$,

$\delta \rho=\left.\delta \rho\right|_{r f}-\rho^{\prime} \delta \tau$.

Thus, one has $\delta \tau=v+B$ and

$$
\begin{aligned}
\delta p_{\text {eff }} & =\left.\delta p_{\text {eff }}\right|_{r f}-p_{\text {eff }}^{\prime} \delta \tau \\
& =c_{s, \text { eff }}^{2} \delta \rho+\delta \rho_{\text {nad }},
\end{aligned}
$$

where $\delta \rho_{\text {nad }}=\left(c_{s, \text { eff }}^{2}-c_{a, \text { eff }}^{2}\right)\left[\delta \rho+\rho^{\prime}(v+B)\right]$ is the intrinsic non-adiabatic perturbation in the fluid. When the fluid is conserved, we have $\bar{\rho}^{\prime}=-3 \mathcal{H}\left(\bar{\rho}+\overline{p_{\text {eff }}}\right)$. By using the relation $\theta=-k^{2}(v+B)$ in Fourier space, one has

$$
\begin{aligned}
\delta p_{\mathrm{eff}} & =c_{s, \text { eff }}^{2} \delta \rho+\left(c_{s, \mathrm{eff}}^{2}-c_{a, \mathrm{eff}}^{2}\right) \rho^{\prime}(v+B) \\
& =c_{s, \mathrm{eff}}^{2} \delta \rho+\left(c_{s, \mathrm{eff}}^{2}-c_{a, \mathrm{eff}}^{2}\right)\left[3 \mathcal{H}\left(\bar{\rho}+\overline{p_{\mathrm{eff}}}\right)\right] \frac{\theta}{k^{2}} .
\end{aligned}
$$

We define the density contrast $\delta=\delta \rho / \bar{\rho}$; then one has the evolution equations for the density perturbation and velocity perturbation for a generic conservation fluid,

$\delta^{\prime}+3 \mathcal{H}\left(c_{s, \text { eff }}^{2}-w_{\text {eff }}\right) \delta+\left(1+w_{\text {eff }}\right)\left(\theta-3 \psi^{\prime}\right)=0$,

$\theta^{\prime}+\mathcal{H}\left(1-3 c_{s, \text { eff }}^{2}\right) \theta-\frac{c_{s, \text { eff }}^{2} k^{2} \delta}{1+w_{\text {eff }}}-k^{2} \phi=0$.

In synchronous gauge, one has

$$
\begin{aligned}
& \phi=\beta^{\prime \prime}+\frac{a^{\prime}}{a} \beta^{\prime}, \\
& \psi=-\frac{h}{6}-\frac{1}{3} \nabla^{2} \beta-\frac{a^{\prime}}{a} \beta^{\prime} .
\end{aligned}
$$

Therefore, $k^{2} \phi=0,-3 \psi^{\prime}=\frac{h^{\prime}}{2}$. Finally, one has the following evolution equations for the density perturbation and the velocity perturbation:

$$
\begin{aligned}
& \delta^{\prime}=-\left(1+w_{\text {eff }}\right)\left(\theta+\frac{h^{\prime}}{2}\right)-3 \mathcal{H}\left(c_{s, \text { eff }}^{2}-w_{\text {eff }}\right) \delta, \\
& \theta^{\prime}=-\mathcal{H}\left(1-3 c_{s, \text { eff }}^{2}\right) \theta+\frac{c_{s, \text { eff }}^{2} k^{2} \delta}{1+w_{\text {eff }}} .
\end{aligned}
$$

Following the formalism for the generalized dark matter case [39], one can recast Eqs. (53) and (54) into

$$
\begin{aligned}
\delta^{\prime}= & -\left(1+w_{\mathrm{eff}}\right)\left(\theta+\frac{h^{\prime}}{2}\right)+\frac{w_{\mathrm{eff}}^{\prime}}{1+w_{\mathrm{eff}}} \delta \\
& -3 \mathcal{H}\left(c_{s, \mathrm{eff}}^{2}-c_{a, \mathrm{eff}}^{2}\right)\left[\delta+3 \mathcal{H}\left(1+w_{\mathrm{eff}}\right) \frac{\theta}{k^{2}}\right], \\
\theta^{\prime}= & -\mathcal{H}\left(1-3 c_{s, \mathrm{eff}}^{2}\right) \theta+\frac{c_{s, \mathrm{eff}}^{2} k^{2} \delta}{1+w_{\mathrm{eff}}},
\end{aligned}
$$

where

$$
\begin{aligned}
& w_{\text {eff }}=-\frac{B_{s}}{B_{s}+\left(1-B_{s}\right) a^{-3(1+\alpha)}}-\sqrt{3} \zeta_{0}, \\
& c_{a, \text { eff }}^{2}=w_{\text {eff }}-\frac{w_{\text {eff }}^{\prime}}{3 \mathcal{H}\left(1+w_{\text {eff }}\right)}, \\
& c_{s, \text { eff }}^{2}=c_{s}^{2}-\frac{\sqrt{3}}{2} \zeta_{0}-\frac{\zeta_{0}}{\sqrt{3} \mathcal{H} \delta}\left(\theta+\frac{h^{\prime}}{2}\right), \\
& c_{s, \text { eff }}^{2}-c_{a, \text { eff }}^{2}=\frac{w_{\text {eff }} \Gamma_{\text {nad,eff }}}{\delta^{\text {rest }}}, \\
& \Gamma_{\text {nad }, \text { eff }}=\frac{\delta p_{\text {nad }}}{p_{\text {eff }}}, \\
& \delta^{\text {rest }}=\delta+3 \mathcal{H}(1+w) \frac{\theta}{k^{2}} .
\end{aligned}
$$

\section{Cosmological constraints from data sets: SNLS3, BAO, Planck, and HST}

In this section, we apply the Markov Chain Monte Carlo method to the investigation of the observational constraints on the viscous generalized Chaplyin gas model which includes bulk viscous perturbations to obtain the parameter space. The MCMC method is based on the publicly available cosmoMC package [40], which has been modified to include the dark fluid perturbation in the CAMB [41] code, which is used to calculate the theoretical CMB power spectrum. To get converging results, in the MCMC calculation we stop sampling by checking the worst e-values [the variance(mean)/mean(variance) of $1 / 2$ chains] and $R-1$ is of the order 0.01. In the following calculations, we take the total likelihood $\mathcal{L} \propto \mathrm{e}^{-\chi^{2} / 2}$ to be the product of the separate likelihoods of SNLS3, BAO, Planck, and HST. Then $\chi^{2}$ is given as

$\chi^{2}=\chi_{\mathrm{SNLS} 3}^{2}+\chi_{\mathrm{BAO}}^{2}+\chi_{\text {Planck }}^{2}+\chi_{\mathrm{HST}}^{2}$,

with the following 8-dimensional parameter space:

$$
P \equiv\left(\omega_{b}, 100 \theta_{\mathrm{MC}}, \tau, \alpha, B_{s}, \zeta_{0}, n_{s}, \log \left[10^{10} A_{s}\right]\right) .
$$


Table 1 The mean values of the model parameters with $1 \sigma, 2 \sigma$ and $3 \sigma$ errors from the combination SNLS3+BAO+Planck+HST

\begin{tabular}{|c|c|}
\hline Model parameters & Mean value with errors \\
\hline$\Omega_{b} h^{2}$ & $0.0222_{-0.000303-0.000590-0.000781}^{+0.000302+0.000603+0.000802}$ \\
\hline $100 \theta_{\mathrm{MC}}$ & $1.051_{-0.000558-0.00110-0.00143}^{+0.000553+0.00109+0.00144}$ \\
\hline$\tau$ & $0.0854_{-0.01354-0.0238-0.0309}^{+0.0121+0.0259+0.0347}$ \\
\hline$\alpha$ & $0.192_{-0.134-0.192-0.192}^{+0.0835+0.195+0.292}$ \\
\hline Bs & $0.808_{-0.0334-0.0624-0.0710}^{+0.0328+0.0629+0.0807}$ \\
\hline$\zeta_{0}$ & $0.0000138_{-0.0000105-0.0000138-0.0000138}^{+0.00000614+0.0000145+0.0000212}$ \\
\hline$n_{s}$ & $0.964_{-0.00710-0.0138-0.0181}^{+0.00714+0.0141+0.0185}$ \\
\hline $\log \left(10^{10} A_{s}\right)$ & $3.0820_{-0.0262-0.0470-0.0615}^{+0.0238+0.0502+0.0660}$ \\
\hline$\Omega_{\mathrm{VGCG}}$ & $0.955_{-0.00173-0.00322-0.00413}^{+0.00172+0.00331+0.00422}$ \\
\hline$\Omega_{b}$ & $0.0453_{-0.00171-0.00331-0.00422}^{+0.00173+0.00322+0.00413}$ \\
\hline$z_{\mathrm{re}}$ & $10.626_{-1.0813-2.159-2.900}^{+1.101+2.172+2.834}$ \\
\hline$H_{0}$ & $71.0621_{-1.349-2.357-3.0527}^{+1.202+2.504+3.287}$ \\
\hline Age/Gyr & $13.723_{-0.0397-0.0791-0.106}^{+0.0395+0.0797+0.103}$ \\
\hline
\end{tabular}

The pivot scale of the initial scalar power spectrum $k_{s 0}=$ $0.05 \mathrm{Mpc}^{-1}$ is used and the priors to the model parameters are taken as follows: the physical baryon density $\omega_{b}(=$ $\left.\Omega_{b} h^{2}\right) \in[0.005,0.1]$; the ratio of the sound horizon and angular diameter distance $100 \theta_{\mathrm{MC}} \in[0.5,10]$; the optical depth $\tau \in[0.01,0.8]$; the model parameters $\alpha \in[0,0.1]$, $B_{s} \in[0,1]$ and $\zeta_{0} \in[0,0.01]$; the scalar spectral index $n_{s} \in[0.5,1.5]$, and the logarithm of the amplitude of the initial power spectrum $\log \left[10^{10} A_{s}\right] \in[2.7,4]$. In addition, the hard coded prior on the comic age 10Gyr $<t_{0}<20 \mathrm{Gyr}$ is imposed. Also, the weak Gaussian prior on the physical baryon density $\omega_{b}=0.022 \pm 0.002$ [42] from big bang nucleosynthesis and the new Hubble constant $H_{0}=$ $74.2 \pm 3.6 \mathrm{~km} \mathrm{~s}^{-1} \mathrm{Mpc}^{-1}$ [43] are adopted. Notice that the current dimensionless energy density of VGCG $\Omega_{\mathrm{VGCG}}$ is not included in the model parameter space $P$, because it is a derived parameter in a spatially flat $(k=0)$ FRW universe. To study the evolution of the perturbation, we should fix the background evolution. To realize that, we use the cosmic observations from the type Ia supernovae SNLS3, cosmic
Fig. 1 The 1D marginalized distribution on individual parameter and 2D contours with $68 \%$ C.L., $95 \%$ C.L., and $99 \%$ C.L. by using SNLS3+BAO+Planck+HST data points

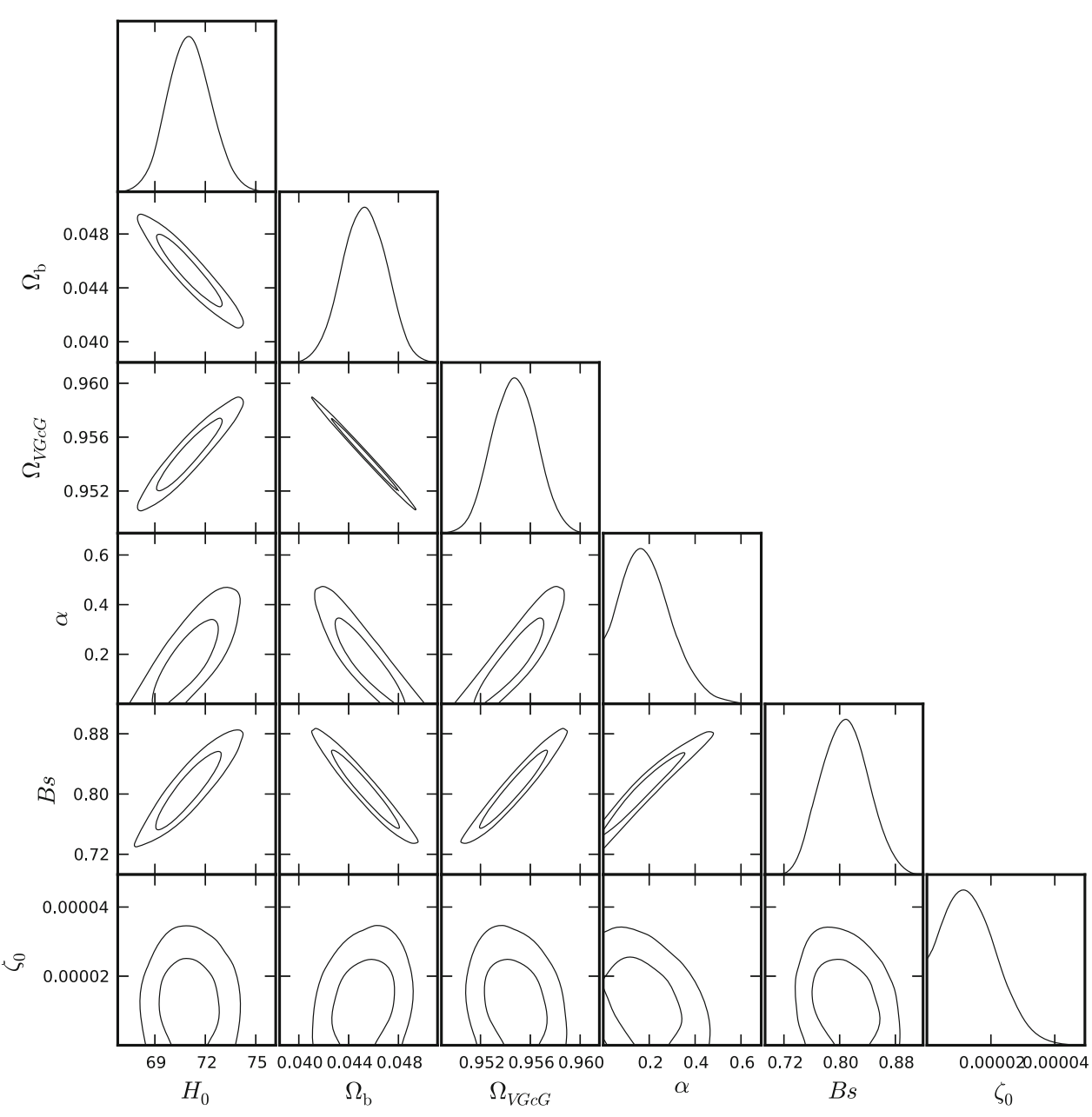




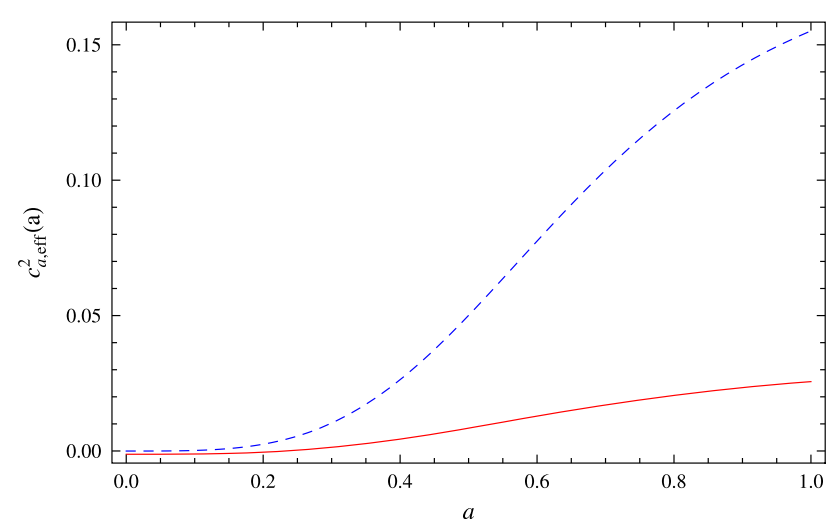

Fig. 2 The evolution of $c_{a \text {,eff }}^{2}$ with respect to the scale factor $a$. The solid curve corresponds to the VGCG2 model (including bulk viscosity perturbations); the dashed curve corresponds to the VGCG1 model (not considering bulk viscosity perturbations)

microwave background radiation from the recently released Planck, baryon acoustic oscillation from the Sloan Digital Sky Survey and the WiggleZ data points and High-redshift SN observations from Hubble Space Telescope. For the detailed description, please see Refs. [44-52].

The best fitting values of the cosmological parameters and the mean values of model parameters with $1 \sigma, 2 \sigma$ and $3 \sigma$ regions in $\mathrm{VGCG}$ model from the combination SNLS3+BAO+Planck+HST are listed in Table 1. Correspondingly, the contour plots are shown in Fig. 1. We find that the minimum $\chi^{2}$ is $\chi_{\min }^{2}=5,115.878$. From Table 1 and Fig. 1, we obtain the constraint on the bulk viscosity

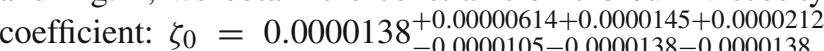
in the $1,2,3 \sigma$ regions, respectively, and it is obvious that we obtain a tighter constraint than our previous results in [27] due to the bulk viscosity perturbation being included. From [27], we know that the value of the bulk viscosity impacts the CMB power spectrum as regards its height of the peak sensitively. Since the parameter $\zeta_{0}$ is related to the dimensionless density parameter of the effective cold dark matter $\Omega_{c 0}$, decreasing the values of $\zeta_{0}$ is equivalent to increasing the value of the effective dimensionless energy density of cold dark matter, so the smaller bulk viscosity $\zeta_{0}$ will make the equality of matter and radiation earlier, therefore the sound horizon is decreased; this can be embodied in the CMB anisotropic power spectra by showing that the first peak is depressed as observed in Fig. 2 in [27].

To show the effect of the bulk viscosity perturbation to the efficient state parameter $w_{\text {eff }}$ and the efficient adiabatic sound speed $c_{a \text {,eff }}^{2}$, we plot the evolution curves of $c_{a \text {, eff }}^{2}$ and $w_{\text {eff }}$ with respect to the scale factor $a$ in Figs. 2 and 3, respectively, which correspond to the VGCG1 model (not considering bulk viscosity perturbations) and the VGCG2 model (including bulk viscosity perturbations). From Fig. 2, one can conclude that the VGCG2 model provides a smaller efficient adiabatic sound speed (which approximately is equal to zero) than the VGCG1 model. It is well known that an almost-zero adiabatic sound speed, which is characterized by the perturbation of density contrast, is important for large scale structure formation. So, the VGCG2 model makes it possible to form large scale structures in our universe. From the upper panel of Fig. 3, one can see that the two VGCG models behave like cold dark matter with almost zero EoS at an early epoch $(a<0.2)$, and they behave like dark energy with EoS $w_{\text {eff }}<0$ at late times, which pushes the universe into an accelerated phase. Furthermore, from the lower panel of Fig. 3, which enlarges the upper panel (from $a=2$ to the end), we can conclude that the VGCG1 model behaves like quintessence $\left(w_{\text {eff }}>-1\right)$ at present, and it behaves phantom-like $\left(w_{\text {eff }}<-1\right)$ in the distant future. However, unlike the VGCG1 model, the VGCG2 model behaves like quintessence at present and in the distant future, which will avoid termination of our universe by a cosmic doomsday. Therefore, it is more necessary and reasonable to include
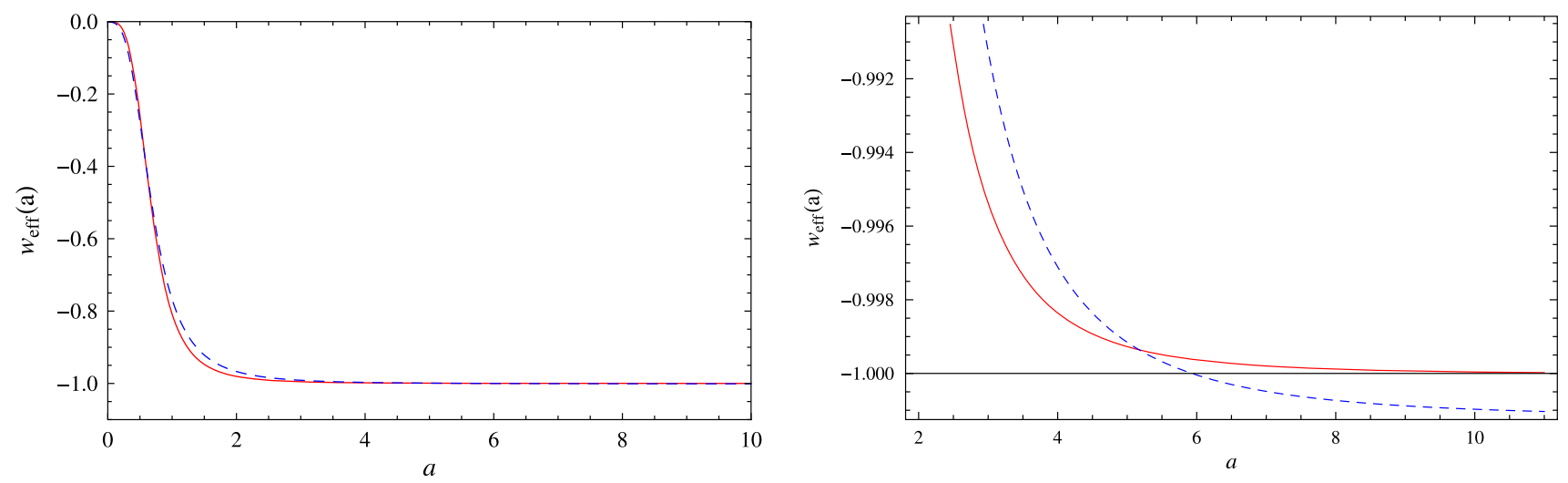

Fig. 3 The evolution of $w_{\text {eff }}$ with respect to scale factor $a$. The solid curve corresponds to theVGCG2 model (including bulk viscosity perturbations); the dashed curve corresponds to the VGCG1 model (not considering bulk viscosity perturbations) 
perturbations of the bulk viscosity when we study cosmic evolution. In conclusion, the VGCG2 model (including bulk viscosity perturbations) as proposed here is a more competitive model than the one we studied previously.

\section{Discussion and conclusion}

In this paper, we have revisited the viscous generalized Chaplygin gas (VGCG) model by including perturbations of the bulk viscosity. We derived the cosmological evolution equations for density and velocity perturbations. By using the MCMC method in combination with SNLS3, BAO, HST and the recently released Planck data points, we obtained tighter constraints as shown in Sect. 4 of this paper. Since the parameter $\zeta_{0}$ is related to the dimensionless density parameter of effective cold dark matter, decreasing the values of $\zeta_{0}$ is equivalent to increasing the value of the effective dimensionless energy density of cold dark matter; this will make the equality of matter and radiation earlier, therefore the sound horizon is decreased. So we predict that the smaller bulk viscosity coefficient parameter $\zeta_{0}=0.0000138_{-0.0000105-0.0000138-0.0000138}^{+0.0000014+0.0000145}$ in the $1,2,3 \sigma$ regions, respectively, will depress the peak of the decreases of $\mathrm{CMB} C_{l}^{\mathrm{TT}}$ power spectrum as regards its height. From Fig. 2, one can conclude that the VGCG2 model provides a smaller efficient adiabatic sound speed than the VGCG1 model, which is important for large scale structure formation. So, the VGCG2 model makes it possible to form large scale structures in our universe. From Fig. 3, one can see that the two VGCG models behave like cold dark matter with almost zero EoS at early epoch $(a<0.2)$, and they behave like dark energy with EoS $w_{\text {eff }}<0$ at late times, which pushes the universe into an accelerated phase. Furthermore, we can see that the VGCG1 model behaves like quintessence $\left(w_{\text {eff }}>-1\right)$ at present, and it behaves phantom-like $\left(w_{\text {eff }}<-1\right)$ in the distant future. However, unlike the VGCG1 model, the VGCG2 model behaves like quintessence at present and in the distant future, which will avoid termination of our universe by a cosmic doomsday. Therefore, it is more reasonable to include perturbations of the bulk viscosity when we study cosmic evolution. Because of the almost-zero sound speed and the almost-negative state parameter (in the distant future), we come to the conclusion that the viscous generalized Chaplygin gas model which includes bulk viscosity perturbations is a competitive alternative for the $\Lambda$ CDM model.

Acknowledgments L. Xu's work is supported in part by NSFC under the Grants No. 11275035 and 'the Fundamental Research Funds for the Central Universities' under Grant No. DUT13LK01.

Open Access This article is distributed under the terms of the Creative Commons Attribution License which permits any use, distribution, and reproduction in any medium, provided the original author(s) and the source are credited.

Funded by $\mathrm{SCOAP}^{3}$ / License Version CC BY 4.0.

\section{References}

1. A.G. Riess et al., Astron. J. 116, 1009 (1998)

2. S. Perlmutter et al., Astrophys. J. 517, 565 (1999)

3. J.L. Tonry et al., Astrophys. J. 594, 1 (2003)

4. A.G. Riess, Astrophys. J. 607, 665 (2004)

5. P. Astier et al., Astron. Astrophys. 447, 31 (2006)

6. D.N. Spergel et al., Astrophys. J. Suppl. Ser. 170, 377 (2007)

7. M. Tegmark et al., Phys. Rev. D 69, 103501 (2004)

8. AYu. Kamenshchik, U. Moschella, V. Pasquier, Phys. Lett. B 511, $265(2001)$

9. V. Gorini, A. Kamenshchik, U. Moschella, Phys. Rev. D 67, 063509 (2003)

10. V. Gorini, A. Kamenshchik, U. Moschella, V. Pasquier, A. Starobinsky, Phys. Rev. D 72, 103518 (2005)

11. AYu. Kamenshchik, U. Moschella, V. Pasquier, Phys. Lett. B 511, $265(2001)$

12. M.C. Bento, O. Bertolami, A.A. Sen, Phys. Rev. D 66, 043507 (2002)

13. J.C. Fabris, S.V.B. Goncalves, P.E. de Souza, Gen. Relativ. Gravit. 34, 53 (2002)

14. V. Gorini, A. Kamenshchik, U. Moschella, V. Pasquier, arXiv: gr-qc/0403062

15. H. Sandvik, M. Tegmark, M. Zaldarriaga, I. Waga, Phys. Rev. D 69, $123524(2004)$

16. R.R.R. Reis, I. Waga, M.O. Calväo, S.E. Joràs, Phys. Rev. D 68, $061302(2003)$

17. W. Zimdahl, J.C. Fabris, Class. Quant. Grav. 22, 4311 (2005)

18. R. Colistete, J. Fabris, J. Tossa, W. Zimdahl, Bulk viscous cosmology. Phys. Rev. D76, 103516 (2007). [arXiv:0706.4086]

19. J.C. Fabris, S.V.B. Goncalves, R. de Sa Ribeiro, Gen. Relativ. Gravit. 38, 495 (2006)

20. V. Gorini, A.Y. Kamenshchik, U. Moschella, O.F. Piatella, A.A. Starobinsky, J. Cosmol. Astropart. Phys. 02, 016 (2008)

21. J.C. Fabris, S.V.B. Goncalves, H.E.S. Velten, W. Zimdahl, Phys. Rev. D 78, 103523 (2008)

22. B. Li, J.D. Barrow, Does bulk viscosity create a viable unified dark matter model? Phys. Rev. D79, 103521 (2009). [arXiv:0902.3163]

23. E. M. Lifshitz, On the gravitational stability of the expanding universe. J. Phys. (USSR) 10, 116 (1946)

24. E.M. Lifshitz, I.M. Khalatnikov, Investigations in relativistic cosmology, Adv. Phys. 12, 185 (1963)

25. J.M. Bardeen, Gauge-invariant cosmological perturbation. Phys. Rev. D 22, 1882 (1980)

26. V.F. Mukhanov, H.A. Feldman, R.H. Brandenberger, Theory of cosmological perturbation. Phys. Rep. 215, 205 (1992)

27. Wei Li, Lixin $\mathrm{Xu}$ Viscous generalized Chaplygin gas as a unified dark fluid. Eur. Phys. J. C 73, 2471 (2013). doi:10.1140/epjc/ s10052-013-2471-1

28. A.B. Balakin, D. Pavon, D.J. Schwarz, W. Zimdahl, New J. Phys. 5, 85 (2003)

29. W. Zimdahl, D.J. Schwarz, A.B. Balakin, D. Pavon, Phys. Rev. D 64, 063501 (2001)

30. R.R.R. Reis, I. Waga, M.O. Calvao, S.E. Joras, Entropy perturbations in quartessence Chaplygin models. Phys. Rev. D 68, 061302 (2003)

31. L. Amendola, I. Waga, F. Finelli, Observational constraint on silent quartessence, 2005. J. Cosmol. Astropart. Phys. JCAP 11, 009 (2005)

32. G. Hinshaw et al., arXiv:1212.5226 (2012)

33. Gong-Bo Zhao et al., Phys. Rev. Lett. 109, 171301 (2012)

34. C. Eckart, Phys. Rev. D58, 919 (1940) 
35. J.-S. Gagnon, J. Lesgourgues, arXiv:1107.1503v2 [astro-ph.CO]

36. Ø. Grøn, Astrophys. Space Sci. 173, 191 (1990)

37. C.P. Ma, E. Bertschinger, Astrophys. J. 455, 7 (1995)

38. J. Hwang, H. Noh, Phys. Rev. D 65, 023512 (2001)

39. W. Hu, Astrophys. J. 506, 485 (1998)

40. A. Lewis, S. Bridle, Phys. Rev. D 66, 103511 (2002). http:// cosmologist.info/cosmomc/

41. http://camb.info/

42. S. Burles, K.M. Nollett, M.S. Turner, Astrophys. J. 552, L1 (2001)

43. A.G. Riess et al., Astrophys. J. 699, 539 (2009)
44. L. Xu, J. Lu, Y. Wang, Eur. Phys. J. C 72, 1883 (2012)

45. L. Xu, arXiv: 1210.5327 [astro-ph.CO]

46. L. Xu, arXiv:1204.5571v1 [astro-ph.CO]

47. L. Xu, arXiv:1208.3715v2 [astro-ph.CO]

48. L. Xu, Y. Wang, H. Noh, Phys. Rev. D 85, 043003 (2012). doi:10. 1103/PhysRevD.85.043003. [arXiv:1112.3701]

49. L. Xu, Y. Wang, H. Noh, Phys. Rev. D. 84, 123004 (2011)

50. J. Valiviita, E. Majerotto, R. Maartens, JCAP 020, 0807 (2008)

51. L. Xu, Y. Wang, JCAP 06, 002 (2010)

52. L. Xu, Y. Wang, Phys. Rev. D 82, 043503 (2010) 